\title{
TMT-based Proteomic Analysis and Protein Expression During Excystation of Cryptosporidium Anderson
}

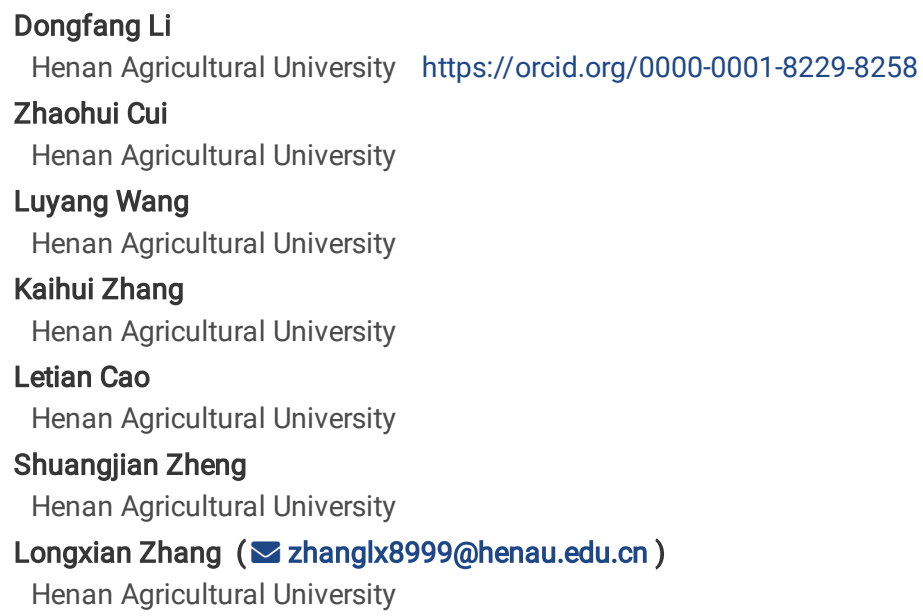




\section{Abstract}

Background: Cryptosporidium andersoni (C. andersoni) initiates infection by the release of sporozoites through excystation. However, the proteins involved in excystation remain unknown. Researching the proteins that participate in the excystation of $C$. andersoni oocysts will fill the gap in our understanding of the excystation system of this parasitic pathogen.

Methods: In this study, C. andersoni oocysts were collected and purified from the feces of naturally infected adult cows. Tandem mass tags (TMT) coupled with liquid chromatograph- tandem mass spectrometry (LC-MS/MS) proteomic analysis was used to investigate the proteomic expression profile of $C$. andersoni oocysts during excystation.

Results: Our proteomic analysis identified a total of 1586 proteins, of which 17 were identified as differentially expressed proteins (DEPs), with 10 upregulated and 7 downregulated proteins. Each of those 17 proteins had multiple biological functions associated with control of gene expression at the level of transcription and biosynthetic and metabolic processes. Quantitative real-time PCR of eight selected genes validated the proteomic data.

Conclusions: Our findings provide new information on the protein composition of $C$. andersoni oocysts as well as possible excystation factors. These data may help us to better understand the pathology of $C$. andersoni and thus may be useful in diagnosis, vaccine development, and immunotherapy for Cryptosporidium.

\section{Background}

Cryptosporidium is an obligate gastrointestinal apicomplexan protozoan parasite that infects all terrestrial organisms, including humans, and most aquatic organisms [1]. Immunosuppressed individuals, particularly acquired immune deficiency syndrome patients with a late diagnosis or without access to highly active antiretroviral therapy, are extremely susceptible to Cryptosporidium infection [2]. There is growing recognition that the parasites can cause both sporadic episodes of illness and far-reaching foodborne or waterborne outbreaks [3]. More importantly, there are no effective treatments or vaccines for cryptosporidiosis at present [2]. Nitazoxanide has been approved by the Food and Drug Administration (FDA) as a treatment drug for cryptosporidiosis, but it has not been demonstrated to be effective in malnourished children or immunosuppressed patients, the most vulnerable population [4]. Cryptosporidium andersoni (C. andersoni) was thought to be large oocysts of Cryptosporidium muris in early reports [5]. In 2000, $C$. andersoni was identified as a novel Cryptosporidium species using morphological, biological, and molecular methods [6]. $C$. andersoni infection can cause different degrees of weight loss, low feed utilization rate, and decreased milk yield in cows, thereby seriously restricting the development of animal husbandry $[7,8]$. In addition, research has shown that $C$. andersoni is the dominant species infecting weaned calves and adult cattle in China [9].

The life-cycle of Cryptosporidium begins with the ingestion of sporulated oocysts by a susceptible host. The sporulated oocysts travel through the digestive tract, excyst in the gastrointestinal tract, and release infective sporozoites that invade the epithelium of the stomach or small intestine [1]. Within host cells, Cryptosporidium undergoes successive rounds of asexual and sexual reproduction, where new oocysts are formed and shed with the feces [10]. The mechanism of excystation, a key step in the infection of Cryptosporidium, is still poorly understood. Cryptosporidium parvum (C. parvum) has been studied in an in vitro excystation scheme that simulated the signals sent by the host, including temperature $\left(37^{\circ} \mathrm{C}\right)$, $\mathrm{pH}$, and the presence of cholate, reducing agents, and proteases $[11,12]$. Unlike $C$. parvum, the excystation of $C$. andersoni oocysts requires only a single temperature stimulus, indicating that the mediating factors of $C$. andersoni excystation may be simpler [13].

Proteomics have been analyzed during sporozoite excystation of Cryptosporidium parvum, and the results have increased our understanding of Cryptosporidium biology [14]. However, it remains to be determined whether a different response is solicited when excystation occurs in other Cryptosporidium species such as Cryptosporidium andersoni infecting the abomasum of cattle [6]. In this study, the proteomics of non-excysted and excysted cow-derived $C$. andersoni oocysts were compared in order to screen proteins associated with excystation and to find potential targets for anticryptosporidiosis.

\section{Methods}

\section{Cryptosporidium andersoni oocyst preparation and excystation}

C. andersoni oocysts were collected and purified from the feces of naturally infected adult cows (without other pathogenic microorganisms) according to previous reports with appropriate modification [15]. In brief, feces of cows were washed with phosphate buffered saline (PBS) three times, and a preliminary purification using Sheather's sugar flotation method was used to remove impurities (incompletely digested silage), followed by further purification using a modified cesium chloride gradient centrifugation to obtain pure oocysts. Purified $C$. andersoni oocysts were counted using a Hemocytometer and stored at $4^{\circ} \mathrm{C}$ in PBS ( $\left.\mathrm{pH} 7.2\right)$ for no longer than two weeks. Oocysts and sporozoites of $C$. andersoni were examined using differential interference contrast (DIC) microscopy (OLYMPUS-BL53, Tokyo, Japan). The specimens for scanning electron microscopy (SEM) were fixed overnight at $4^{\circ} \mathrm{C}$ in $2.5 \%$ glutaraldehyde in $0.1 \mathrm{M}$ phosphate buffer and then washed two times for 15 min with the same buffer. After dehydration in a graded ethanol series, the ethanol was replaced with isoamyl acetate twice for $20 \mathrm{~min}$, after which the specimens were dried using the critical point technique, coated with gold, and examined using a Hitachi s-3400N SEM (HITACHI, Japan). A total of $1.8 \times 10^{9} \mathrm{C}$. andersoni oocysts were used in the proteomics analyses; each group (excysted group and non-excysted group) used $9 \times 10^{8}$ oocysts, with three independent biological replicates ( $3 \times$ $10^{8}$ each). Before the experiments, oocysts were subjected to $2.5 \%$ sodium hypochlorite solution for 10 min at $4^{\circ} \mathrm{C}$ and washed three times with PBS. 
Excystation was performed at $37^{\circ} \mathrm{C}(\sim 3 \mathrm{~h}$ and mixing every $10 \mathrm{~min})$ and continued until $>80 \%$ excystation had been observed by microscopic examination at $\times 400$ magnification [13].

\section{Protein extraction and SDS-PAGE separation}

Lysis buffer ( $\mathrm{pH}$ 8.5) containing $7 \mathrm{M}$ urea, $2 \mathrm{M}$ thiourea, $65 \mathrm{mM}$ Tris, 2\% Dithiothreitol (DTT), 4\% 3-[(3-Cholamidopropyl)dimethylammonio]-1propanesulfonate (CHAPS), $0.2 \%$ IPG buffer (GE Amersham, USA), and $0.1 \%$ v/v protease inhibitor cocktail (Merck, USA) was added into the tubes containing excysted or unexcysted oocysts [14]. Oocyst disruption was achieved by sonication at $80 \mathrm{~W}$ for $3 \mathrm{~s} \times 100$ at intervals of $10 \mathrm{~s}$. The debris was removed by centrifugation at $12,000 \mathrm{~g}$ at $4^{\circ} \mathrm{C}$ for $10 \mathrm{~min}$; the supernatant was transferred to a new centrifuge tube, and the concentration was determined using a BCA Protein Assay kit (Beyotime Biotechnology, China) according to the manufacturer's instructions. The oocysts were stored at $-80^{\circ} \mathrm{C}$ until the following proteomic analyses.

\section{Trypsin Digestion, TMT Labeling, and HPLC Fractionation}

TMT tagging and analysis were performed as described previously [15]. For digestion, the protein solution was reduced with $5 \mathrm{mM}$ dithiothreitol for 30 min at $56^{\circ} \mathrm{C}$ and alkylated with $11 \mathrm{mM}$ iodoacetamide for $15 \mathrm{~min}$ at room temperature in darkness. The protein sample was then diluted by adding 100 $\mathrm{mM}$ Triethylamonium bicarbonat (TEAB) to urea at a concentration less than $2 \mathrm{M}$. Finally, trypsin was added at a 1:50 trypsin-to-protein mass ratio for the first digestion overnight and at a 1:100 trypsin-to-protein mass ratio for a second 4-h digestion. After trypsin digestion, the peptides were desalted using a Strata X C18 SPE column (Phenomenex) and vacuum-dried. Peptides were reconstituted in $0.5 \mathrm{M}$ TEAB and processed according to the manufacturer's protocol for a TMT kit. Briefly, one unit of TMT reagent was thawed and reconstituted in acetonitrile. The peptide mixtures were then incubated for $2 \mathrm{~h}$ at room temperature and pooled, desalted, and dried by vacuum centrifugation. The tryptic peptides were fractionated by high pH reverse-phase HPLC using an Agilent 300 Extend C18 column ( $5 \mu \mathrm{m}$ particles, $4.6 \mathrm{~mm}$ ID, $250 \mathrm{~mm}$ length). Briefly, peptides were first separated into 60 fractions with a gradient of $8 \%-32 \%$ acetonitrile $(\mathrm{pH} 9.0)$ over $60 \mathrm{~min}$. Then, the peptides were combined into 18 fractions and dried by vacuum centrifuging.

\section{LC-MS/MS analysis and Database Search}

The tryptic peptides were dissolved in $0.1 \%$ formic acid (solvent $\mathrm{A}$ ) and directly loaded onto a home-made reversed-phase analytical column (15-cm length, $75 \mu \mathrm{m}$ i.d.). The gradient comprised an increase from $6 \%$ to $23 \%$ solvent B $(0.1 \%$ formic acid in $98 \%$ acetonitrile) over $26 \mathrm{~min}, 23 \%-35 \%$ in $8 \mathrm{~min}$, increasing to $80 \%$ in $3 \mathrm{~min}$, and then holding at $80 \%$ for the last $3 \mathrm{~min}$, all at a constant flow rate of $400 \mathrm{~nL} / \mathrm{min}$ on an EASY-nLC 1000 UPLC system [16].

The peptides were subjected to an NSI source, followed by tandem mass spectrometry (MS/MS) in a Q ExactiveTM Plus (Thermo) coupled online to the UPLC. The electrospray voltage applied was $2.0 \mathrm{kV}$. The $\mathrm{m} / \mathrm{z}$ scan range was 350-1800 for full scan, and intact peptides were detected in the Orbitrap at a resolution of 70,000. Peptides were then selected for MS/MS using the NCE setting of 28 , and the fragments were detected in the Orbitrap at a resolution of 17,500. A data-dependent procedure that alternated between one MS scan followed by 20 MS/MS scans with 15.0 s dynamic exclusion was used. Automatic gain control (AGC) was set at 5E4. The fixed first mass was set as $100 \mathrm{~m} / \mathrm{z}$ [17].

The resulting MS/MS data were processed using the Maxquant search engine (v.1.5.2.8). Tandem mass spectra were searched against the Proteome Cryptosporidium database (28217 sequences) concatenated with the reverse decoy database. Trypsin/P was specified as a cleavage enzyme, allowing up to two missing cleavages. The mass tolerance for precursor ions was set as 20 ppm in the First search and 5 ppm in the Main search, and the mass tolerance for fragment ions was set as $0.02 \mathrm{Da}$. Carbamidomethyl on Cys was specified as a fixed modification, and oxidation on Met was specified as a variable modification. The FDR was adjusted to $<1 \%$, and the minimum score for peptides was set as $>40$.

\section{Bioinformatic analysis}

Multiple bioinformatics tools were employed to analyze the proteins. Gene Ontology (GO) annotation of the proteome was derived from the UniProt-GOA database (http://www.ebi.ac.uk/GOA/) that classified proteins into three categories: biological process, cellular compartment, and molecular function. The Clusters of orthologous groups for eukaryotic complete (KOG) database was used for functional classification statistics of differentially expressed proteins (DEPs). The Kyoto Encyclopedia of Genes and Genomes (KEGG) database was used to annotate protein pathways. For GO, KOG, and KEGG enrichment analyses, a two-tailed Fisher's exact test was applied to test DEPs against all identified proteins, and a corrected $p$ value $<0.05$ was considered significant. Identified protein domain functional descriptions were annotated by InterProScan (a sequence analysis application) based on the protein sequence alignment method, and the InterPro domain database (http://www.ebi.ac.uk/interpro/) was used. We used Wolfpsort, a subcellular localization prediction program (https://www.genscript.com/wolf-psort.html) to predict subcellular localization. Wolfpsort is an updated version of PSORT/PSORT II for the prediction of eukaryotic sequences. 
The qRT-PCR was used to determine and verify gene expression levels of eight DEPs in the excystation of $C$. andersoni oocyst [18]. Total RNA of each sample was extracted from excysted and unexcysted $C$. andersoni oocysts (with three biological replicates for each group) using TRIzol ${ }^{\mathrm{TM}}$ Reagent (Invitrogen, USA). RNA purification and reverse transcription were performed using a Reverse Transcriptase M-MLV Kit with gDNA Eraser (Takara, Japan) according to the manufacturer's instructions. The quantity of RNA was analyzed using a Nano Drop One (Thermo Scientific Fisher, US). For every sample, $1 \mu \mathrm{g}$ of total RNA was treated with $1 \mu \mathrm{L}$ of gDNA Eraser at $42^{\circ} \mathrm{C}$ for 2 min. First-strand cDNA was synthesized using $1 \mu \mathrm{L}$ of Oliga(dT) and $1 \mu \mathrm{L}$ of Random Primers and RNase-free $\mathrm{dH}_{2} \mathrm{O}$ (up to $10 \mu \mathrm{L}$ ) at $70^{\circ} \mathrm{C}$ for $10 \mathrm{~min}$ and at $4^{\circ} \mathrm{C}$ for $2 \mathrm{~min}$. Second strand cDNA was synthesized using $4 \mu \mathrm{L}$ of $5 \times \mathrm{M}$ MLV buffer, $1 \mu \mathrm{L}$ of dNTP, $0.5 \mu \mathrm{L}$ of RI, $0.5 \mu \mathrm{L}$ of M-MLV, $4 \mu \mathrm{L}$ RNase-free $\mathrm{dH}_{2} \mathrm{O}$, and $10 \mu \mathrm{L}$ of first-strand cDNA. The resulting products were used as templates for qRT-PCR. Gene-specific qRT-PCR primers were designed with Premier 5.0 software (Premier Biosoft International, Palo Alto, CA, USA). Oligonuleotide sequences of target and reference genes (18s) for qRT-PCR are listed in Supplementary Table S1. The qRT-PCR reaction was composed of $2 \mu \mathrm{L}$ of cDNA, $0.4 \mu \mathrm{M}$ (final concentration) of each primer, and $5 \mu \mathrm{L}$ of $2 \times$ SYBR qPCR mix (Takara, Japan). PCR reactions were performed in duplicate using the qTOWER ${ }^{3} \mathrm{G}$ IVD (Analytik Jena AG, Germany) with the following cycles: one cycle for denaturing at $95^{\circ} \mathrm{C}$ for $30 \mathrm{~s}, 40$ cycles for PCR reaction at $95^{\circ} \mathrm{C}$ for $5 \mathrm{~s}, 55^{\circ} \mathrm{C}$ for $10 \mathrm{~s}$, and $72^{\circ} \mathrm{C}$ for $15 \mathrm{~s}$. One cycle of melting curve for all reactions was added to verify product specificity, with $95^{\circ} \mathrm{C}$ for $15 \mathrm{~s}$ and $65^{\circ} \mathrm{C}$ for $60 \mathrm{~s}$. Relative expression levels were normalized to a housekeeping gene, $18 \mathrm{~s}$ RNA. The $2^{-\Delta \Delta C T}$ method was used to determine the fold change of gene expression levels. GraphPad Prism V 8.0 (https://www.graphpad.com/) was used to analyze and plot the data.

\section{Results}

\section{Purification and excystation of Cryptosporidium andersoni oocysts}

Highly purified oocysts of $C$. andersoni were obtained from adult cow's feces via sucrose solution density gradient centrifugation and cesium chloride density gradient centrifugation (Figure 2). The excystation rate of $C$. andersoni was $82 \%$ using $3-$ h incubation in a $37^{\circ} \mathrm{C}$ thermostat water bath. Nonexcysted oocysts, excysted oocysts, and sporozoites are shown in Figure 3. Most oocysts were round or oval, with a transparent wall around the oocyst, a bright vestigial body, and four sporozoites inside the oocyst. After excystation, the sporozoites swim snakelike or oscillate violently, and residual bodies either remain in the oocyst or prolapse outside the oocyst wall.

\section{Protein identification and quantification}

To understand how the proteome changes with Cryptosporidium andersoni oocyst excystation, a TMT-based labeling approach coupled with High Performance Liquid Chromatography (HPLC) and LC-MS/MS was employed to identify the proteome of excysted and non-excysted oocysts (Fig. 1). A total of 106840 two-stage spectra were obtained by mass spectrometry analysis. A subset of 20541 effective spectra were obtained after the mass spectrometry two-level graph was searched by the protein theory data, and the spectral graph utilization rate was $19.2 \%$. A total of 10789 peptide segments were identified by spectral analysis, with 10213 unique peptide segments, and 1786 proteins were identified (Fig. 4). In addition, most of the matching errors for the majority of the peptides ranged from -5 to $10 \mathrm{ppm}$, indicating the good reliability of the TMT data in this study. The statistical analysis of protein identification is provided in Table S1. Our dataset comprised $46.1 \%$ of the $C$. andersoni predicted proteome (3876). Among those proteins, 44.3\% (791/1786) were uncharacterized proteins, and 4.4\% (78/1786) were ribosomal proteins.

\section{Functional categories of proteins of $C$. andersoni oocysts}

All 1786 proteins were subjected to GO, KOG, and subcellular classification analyses (Table S2). According to the G0 analysis, 1258,696 , and 1122 proteins were assigned to the categories 'biological processes,' 'molecular function,' and 'cellular components,' respectively (Fig. 4). The top five most frequent categories of biological processes in our study were "cellular processes" (415), "metabolic processes" (408), "single-organism processes" (171), "localization" (95), and "biological regulation" (78). To predict and classify their possible functions based on reference to orthologs from other species, all proteins were annotated using the KOG database. As shown in Figure 4, a total of 1107 proteins were categorized into 25 groups, among which "Posttranslational modification, protein turnover, chaperones" accounted for the largest group (191), followed by "Translation, ribosomal structure and biogenesis" (149) and "Intracellular trafficking, secretion, and vesicular transport" (100). Subcellular classification showed that those proteins were localized in the "cytoplasm" (678), "plasma membrane" (278), "extracellular" (230), "nucleus" (215), "mitochondria” (111), "cytoplasm, nucleus" (108), "cytoskeleton" (60), and "endoplasmic reticulum" (40) (Fig. 4).

\section{TMT analysis and identification of differentially expressed proteins}

We used a 1.2-fold increase or decrease in protein expression as a benchmark for physiologically significant changes to determine the proteins that were differentially expressed after excystation. In total, 17 DEPs were identified between excysted and non-excysted oocysts. Among these DEPs, 10 were upregulated and 7 were downregulated after $C$. andersoni excystation (Table S3). According to the GO enrichment analysis (Fig. 5), 13, 8, and 12 proteins were enriched for biological process, cell component, and molecular function, respectively. Subcellular localization predictions showed that the DEPs were 
located in the cytoplasm (4), plasma membrane (3), nucleus (3), extracellular (2), mitochondria (2), cytoplasm or nucleus (1), and cytoskeleton (1) (Fig. 5). Functional enrichment analyses of the DEPs (Fig. 5) suggested that the DEPs were significantly enriched for "RNA processing and modification" (2), "posttranslational modification, protein turnover, chaperones" (2), "general function prediction only" (2), "chromatin structure and dynamics" (1), "energy production and conversion" (1), "amino acid transport and metabolism" (1), "lipid transport and metabolism" (1), and "translation, ribosomal structure and biogenesis" (1). Unfortunately, these proteins were not enriched into any KEGG pathway. In addition, we focused on upregulated proteins after excystation of $C$. andersoni oocysts. The unregulated proteins were significantly enriched in "binding" (3) and were mainly localized in the cytoplasm (3), nucleus (2), and mitochondria (2) (Fig. 6), suggesting that upregulated proteins were mainly associated with control of gene expression at the level of transcription, for example RCC1, Histone H2A, and an unidentified protein (ID: A0A1J4MP75).

\section{Validation of the transcription of DEPs by real-time quantitative PCR}

To investigate the excystation mechanism, the transcription levels of DEPs were verified by qRT-PCR. Nine DEPs were selected randomly for qRT-PCR analysis to characterize gene expression. The qRT-PCR results indicated that the transcription levels of most genes were consistent with expression levels according to TMT data (Fig. 7).

\section{Discussion}

To obtain a better understanding of the molecular mechanisms and proteome changes occurring when $C$. andersoni excysts in vitro, a TMT-based quantitative proteomics analysis was performed to directly screen differentially abundant proteins involved in the excystation of this parasite. In this study, 1786 proteins were identified in the excysted oocyst/sporozoite of $C$. andersoni, approximately $46.1 \%$ of the predicted proteome (3876). The number of identified proteins in the excystation of $C$. andersoni was greater than in previous proteome studies of oocysts and sporozoites of $C$. parvum; in 2007, 303 proteins were identified during sporozoite excystation of C. parvum [19]; in 2008, 1237 nonredundant proteins (approximately $30 \%$ of the predicted proteome) of excysted $C$. parvum oocyst/sporozoite were identified using LC-MS/MS analysis [20], and in 2013, 33 separate $C$. parvum sporozoite proteins were identified from 135 protein hits using SDS-PAGE with subsequent LC-MS/MS analysis [21]. Among the proteins identified in this study, many have been identified as putative virulence factors by immunological and molecular methods [1]; for example, serine protease and aminopeptidase are associated with excystation; P23 and P30 are associated with adhesion; TRAP and thrombospondin-related proteins are involved in parasite gliding motility and cell penetration; Cp2, Cap135, and secretory phospholipase are associated with invasion, and HSP70 and HSP90 are associated with stress protection [1].

Using a comparative proteomic approach, we identified 17 DEPs between the excysted and non-excysted $C$. andersoni oocysts. Further analysis of these DEPs was performed to understand the mechanism of $C$. andersoni excystation, especially the upregulated proteins. Compared with non-excysted $C$. andersoni oocysts, the RCC1 (regulator of chromosome condensation) increased by a factor of about 3.5 in the excysted oocysts. Histone H2A and an unidentified protein (Protein accession: A0A1J4MP75) containing High Mobility group box domains (HMG-box) were also increased after excystation. RCC1 is bound to chromatin and confined to the nucleus. RCC1 ß-propeller domain binds the histone H2A/H2B dimer component of the histone octamer that can regulate the concentration gradient of Ran-GTP around the chromosomes to mediate nucleocytoplasmic transport and mitotic spindle assembly $[22,23,24]$. An RCC1 mutant of Toxoplasma gondii showed defects in nuclear trafficking and growth impairment under nutrient limitation, demonstrating that the rate of nuclear transport is a critical factor affecting growth in low-nutrient conditions [25]. High mobility group box (HMGB) proteins have been reported in many apicomplexan parasites, including Plasmodium falciparum [26], Toxoplasma gondii [27], and Babesia bovis [28]. PfHMGB1 andPfHMGB2 are potent inducers of two important mediators of inflammation, TNFa and iNOS, suggesting that these proteins may have immunomodulatory roles in the pathophysiology of $P$. falciparum infection [26]. TgHMGB1 was implicated in transcriptional regulation and most likely acts as an activator of many virulence factors in T. gondii [27]. Based on the above conclusions, we hypothesized that RCC1, H2A, and the unidentified protein were involved in the gene expression regulation of $C$. andersoni excystation factors.

Phosphatidylethanolamine (PtdEtn) had a 1.258 rate increase after excystation of $C$. andersoni. PtdEtn is located at the plasma membrane and is enriched in a variety of biosynthetic and metabolic processes, including lipid biosynthetic and metabolic processes, organophosphate biosynthesis and metabolism, phospholipid metabolism, and organic substance metabolism. The increase of PtdEtn may be associated with energy acquisition in the excystation of $C$. andersoni oocysts. PtdEtn is one of the most abundant phospholipids in prokaryotes and eukaryotes; it contributes to the membrane integrity, membrane fusion/fission, protein stabilization, and autophagy events [29]. PtdEtn is the second major phospholipid classified in T. gondii. Phosphatidylserine decarboxylase (PSD) mediates the decarboxylation of phosphatidylserine (PtdsSer) to form PtdEtn and displays much higher (10fold) activity in T. gondii tachyzoites compared with yeast and mammalian cells [30]. In addition, choline kinase inhibitors inhibit the ethanolamine kinase activity of $P$. falciparum choline kinase, leading to a severe decrease in the phosphatidylethanolamine levels within $P$. falciparum, which explains the resulting growth phenotype and parasite death [31].

The B6AJJ3 protein is an uncharacterized protein that contains a WD40-repeat-containing domain and a YVTN-type repeat domain. The expression level of the protein increased after excystation of $C$. andersoni oocysts. During the Cryptosporidium life-cycle, sporozoites slip out of the oocyst and infect the host cells, and this mainly occurs in the intestinal lumen or stomach. This uncharacterized protein may be associated with environmental stress and the host immune system. WD40 proteins are much more abundant in eukaryotic organisms, where they participate in a diverse set of functions, including signal transduction, cell division, cytoskeleton assembly, chemotaxis, and RNA processing [32]. The WD40-repeat protein-like protein PfWLP1 may 
support the stability of adhesion protein complexes of the plasmodia blood stages [33]. The YVTN-type repeat domain is found in archaeal surface layer proteins that protect cells from extreme environments [34].

In addition to the DEPs, many known and potential virulence factors were identified in the oocysts of $C$. andersoni. Although there were no significant differences in the expression of those virulence factors in the excysted and non-excysted $C$. andersoni oocysts, their role in sporozoite adhesion and invasion of host cells is still worthy of study. The Cpa135 protein was localized in the apical complex of the sporozoite and in the parasitophorous vacuole (PV) during the intracellular stages. In fact, in the oocyst-sporozoite, both the Cpa135 mRNA and the Cpa135 protein are present, and the protein rapidly increases during the excystation process of $C$. parvum [35]. This study identified a variety of heat shock proteins (HSPs), including HSP10, HSP70, and HSP90. HSPs are involved in maintaining cell homeostasis [36]. Synthesis of HSPs, especially HSP70, increases dramatically under stressful conditions such as a sudden temperature shift, changes in concentrations of glucose and calcium, or in response to immune effectors [37]. Previous studies have identified two HSPs in Cryptosporidium, HSP70 and HSP90 [38, 39]. Differences in HSP expression in T. gondii correlate with parasite virulence in the immunocompetent host [40]. The relationship between the expression levels of these HSPs and Cryptosporidium virulence is worthy of further study.

\section{Conclusion}

TMT-based proteomics technology provides a new method for the identification of proteins involved in the excystation of $C$. andersoni oocysts. The proteomes of excysted and non-excysted $C$. andersoni oocysts were compared, and multiple proteins such as RCCl, $\mathrm{Histone} \mathrm{H} 2 \mathrm{~A}$, and $\mathrm{PtdEtn}$ and two uncharacterized proteins (Protein accession numbers: B6AJJ3 and A0A1J4MP75) had increased expression in the excysted C. andersoni oocysts; these proteins may be key regulatory factors involved in the excystation of $C$. andersoni.

\section{Abbreviations}

TMT: tandem mass tag

HPLC: high performance liquid chromatography

LC-MS/MS: liquid chromatography-mass spectrometry/mass spectrometry

GO: gene ontology

KOG: clusters of orthologous for eukaryotic complete genomes

KEGG: Kyoto encyclopedia of genes and genomes

SDS-PAGE: sodium dodecyl sulfate polyacrylamide gel electrophoresis

PBS: phosphate buffered saline

RT-qPCR: real-time quantitative polymerase chain reaction

PV: parasitophorous vacuole

HSPs: Heat shock proteins

\section{Declarations}

\section{Authors' contributions}

LXZ conceived and designed the experiments. DFL, SJZ, LYW, and LTC collected the specimens. DFL, ZHC, and KHZ performed the experiments. DFL and ZHC analyzed the data. DFL and LXZ participated in drafting and revising the manuscript. All authors have read and approved the final manuscript.

\section{Funding}

This study was supported in part by the National Natural Science Foundation of China (U1904203), the National Key Research and Development Program of China (2017YFD0501305, 2019YFC1605700), and the Leading talents of the Thousand Talents Program of Central China (19CZ0122). The sponsors played no role in the study design, in the collection, analysis, or interpretation of the data, in writing the report, or in the decision to submit the article for publication.

\section{Availability of data and materials}

The datasets supporting the conclusions of this article are included within the article and its additional files. The mass spectrometry proteomics data have been deposited at the ProteomeXchange Consortium via the PRIDE partner repository with the dataset identifier PXD028423. All analyzed data are available from the corresponding author upon reasonable request. 


\section{Ethics statement}

Permission was obtained from animal owners or managers prior to feces specimen collection, and no specific permits were required for the described field studies. The study protocol was reviewed and approved by the Research Ethics Committee of Henan Agricultural University (Approval No. IECHENAU-20170928-06).

\section{Competing interests}

The authors declare that they have no competing interests.

\section{References}

1. Bouzid M, Hunter PR, Chalmers RM, Tyler KM. Cryptosporidium pathogenicity and virulence. Clin Microbiol Rev. 2013;261:115-34; doi: 10.1128 /CMR.00076-12. https://www.ncbi.nlm.nih.gov/pubmed/23297262.

2. Ryan U, Zahedi A, Paparini A. Cryptosporidium in humans and animals-a one health approach to prophylaxis. Parasite Immunol 2016;389:535-47; doi:10.1111/pim.12350. https://www.ncbi.nlm.nih.gov/pubmed/27454991.

3. Zahedi A, Ryan U. Cryptosporidium - An update with an emphasis on foodborne and waterborne transmission. Res Vet Sci. 2020;132:500-12; doi:10.1016/j.rvsc.2020.08.002. https://www.ncbi.nlm.nih.gov/pubmed/32805698.

4. Amadi B, Mwiya M, Sianongo S, Payne L, Watuka A, Katubulushi M, et al. High dose prolonged treatment with nitazoxanide is not effective for cryptosporidiosis in HIV positive Zambian children: a randomised controlled trial. BMC Infect Dis. 2009;9:195. doi:10.1186/1471-2334-9-195.

5. Anderson BC. Abomasal cryptosporidiosis in cattle. Vet Pathol 1987;243:235-8; doi:10.1177/030098588702400307. https://www.ncbi.nlm.nih.gov/pubmed/3603962.

6. Lindsay DS, Upton SJ, Owens DS, Morgan UM, Mead JR, Blagburn BL. Cryptosporidium andersoni n. sp. (Apicomplexa: Cryptosporiidae) from cattle, Bos taurus. J Eukaryot Microbiol 2000;471:91-5; doi:10.1111/j.1550-7408.2000.tb00016.x. https://www.ncbi.nlm.nih.gov/pubmed/10651302.

7. Xiao L. Molecular epidemiology of cryptosporidiosis: an update. Exp Parasitol 2010;1241:80-9; doi:10.1016/j.exppara.2009.03.018. https://www.ncbi.nlm.nih.gov/pubmed/19358845.

8. Paul S, Chandra D, Tewari AK, Banerjee PS, Ray DD, Raina OK, et al. Prevalence of Cryptosporidium andersoni: a molecular epidemiological survey among cattle in India. Veterinary parasitology. 2009;1611-2:31-5. doi:10.1016/j.vetpar.2008.12.013.

9. Wang R, Ma G, Zhao J, Lu Q, Wang H, Zhang L, et al. Cryptosporidium andersoni is the predominant species in post-weaned and adult dairy cattle in China. Parasitology international. 2011;601:1-4. doi:10.1016/j.parint.2010.09.002.

10. Gunasekera S, Zahedi A, O'Dea M, King B, Monis P, Thierry B, et al. Organoids and Bioengineered Intestinal Models: Potential Solutions to the Cryptosporidium Culturing Dilemma. Microorganisms 2020;85; doi:10.3390/microorganisms8050715.

11. Smith HV, Nichols RA, Grimason AM. Cryptosporidium excystation and invasion: getting to the guts of the matter. Trends Parasitol 2005;213:133-42; doi:10.1016/j.pt.2005.01.007. https://www.ncbi.nlm.nih.gov/pubmed/15734661.

12. Robertson LJ, Campbell AT, Smith HV. In vitro excystation of Cryptosporidium parvum. Parasitology. 1993;106 (1):13-9; doi:10.1017/s003118200007476x. https://www.ncbi.nlm.nih.gov/pubmed/8479797.

13. Widmer G, Klein P, Bonilla R. Adaptation of Cryptosporidium oocysts to different excystation conditions. Parasitology. 2007;134(11):1583-8; doi: 10.1017/S0031182007002922. https://www.ncbi.nlm.nih.gov/pubmed/17506930.

14. Wiśniewski JR, Zougman A, Nagaraj N, Mann M. Universal sample preparation method for proteome analysis. Nat Methods. 2009;6(5):359-62. doi:10.1038/nmeth.1322. https://pubmed.ncbi.nIm.nih.gov/19377485.

15. hompson A, Schäfer J, Kuhn K, Kienle S, Schwarz J, Schmidt G, Neumann T, Johnstone R, Mohammed AK, Hamon C. Tandem mass tags: a novel quantification strategy for comparative analysis of complex protein mixtures by MS/MS. Anal Chem. 2003;75(8):1895-904. doi:10.1021/ac0262560. https://pubmed.ncbi.nlm.nih.gov/12713048.

16. Zhang L, Elias JE. Relative Protein Quantification Using Tandem Mass Tag Mass Spectrometry. Methods Mol Biol. 2017;1550:185-98. doi:10.1007/978-1-4939-6747-6_14. https://pubmed.ncbi.nlm.nih.gov/28188531.

17. Domon B, Aebersold R. Mass spectrometry and protein analysis. Science. 2006;312(5771):212-7. doi:10.1126/science.1124619. https://pubmed.ncbi.nlm.nih.gov/16614208.

18. Bajszár G, Dekonenko A. Stress-induced Hsp70 gene expression and inactivation of Cryptosporidium parvum oocysts by chlorine-based oxidants. Appl Environ Microbiol 2010 Mar;76(6):1732-9. doi:10.1128/AEM.02353-09. https://pubmed.ncbi.nlm.nih.gov/20118357.

19. Snelling WJ, Lin Q, Moore JE, Millar BC, Tosini F, Pozio E, et al. Proteomics analysis and protein expression during sporozoite excystation of Cryptosporidium parvum (Coccidia, Apicomplexa). Mol Cell Proteomics. 2007;62:346-55; doi:10.1074/mcp.M600372-MCP200.

https://www.ncbi.nlm.nih.gov/pubmed/17124246.

20. Sanderson SJ, Xia D, Prieto H, Yates J, Heiges M, Kissinger JC, et al. Determining the protein repertoire of Cryptosporidium parvum sporozoites. Proteomics. 2008;87:1398-414; doi:10.1002/pmic.200700804. https://pubmed.ncbi.nlm.nih.gov/18306179/. 
21. Siddiki AZ. Sporozoite proteome analysis of Cryptosporidium parvum by one-dimensional SDS-PAGE and liquid chromatography tandem mass spectrometry. J Vet Sci. 2013;142:107-14; doi:10.4142/jvs.2013.14.2.107. https://www.ncbi.nlm.nih.gov/pubmed/23814469.

22. Clarke PR, Zhang C. Ran GTPase: a master regulator of nuclear structure and function during the eukaryotic cell division cycle? Trends Cell Biol. 2001;11 9:366-71; doi:10.1016/s0962-8924(01)02071-2. https://www.ncbi.nlm.nih.gov/pubmed/11514190.

23. Carazo-Salas RE, Guarguaglini G, Gruss OJ, Segref A, Karsenti E, Mattaj IW. Generation of GTP-bound Ran by RCC1 is required for chromatin-induced mitotic spindle formation. Nature. 1999;400 6740:178-81. doi:10.1038/22133.

24. Makde RD, England JR, Yennawar HP, Tan S. Structure of RCC1 chromatin factor bound to the nucleosome core particle. Nature. 2010;467(7315):562-6; doi:10.1038/nature09321. https://www.ncbi.nlm.nih.gov/pubmed/20739938.

25. Frankel MB, Mordue DG, Knoll LJ. Discovery of parasite virulence genes reveals a unique regulator of chromosome condensation 1 ortholog critical for efficient nuclear trafficking. Proc Natl Acad Sci U S A. 2007;104 24:10181-6; doi:10.1073/pnas.0701893104.

https://www.ncbi.nlm.nih.gov/pubmed/17535896.

26. Kumar K, Singal A, Rizvi MM, Chauhan VS. High mobility group box (HMGB) proteins of Plasmodium falciparum: DNA binding proteins with proinflammatory activity. Parasitol Int. 2008;57(2):150-7; doi:10.1016/j.parint.2007.11.005. https://www.ncbi.nlm.nih.gov/pubmed/18234548.

27. Wang H, Lei T, Liu J, Li M, Nan H, Liu Q. A nuclear factor of high mobility group box protein in Toxoplasma gondii. PLoS One. 2014;9(11):e111993; doi:10.1371/journal.pone.0111993. https://www.ncbi.nlm.nih.gov/pubmed/25369210.

28. Dalrymple BP, Peters JM. Characterization of a cDNA clone from the haemoparasite Babesia bovis encoding a protein containing an "HMG-Box". Biochem Biophys Res Commun. 1992;184(1):31-5; doi:10.1016/0006-291x(92)91153-h. https://www.ncbi.nlm.nih.gov/pubmed/1567437.

29. Vance JE, Tasseva G. Formation and function of phosphatidylserine and phosphatidylethanolamine in mammalian cells. Biochim Biophys Acta. 2013;1831(3):543-54; doi:10.1016/j.bbalip.2012.08.016. https://www.ncbi.nlm.nih.gov/pubmed/22960354.

30. Gupta N, Zahn MM, Coppens I, Joiner KA, Voelker DR. Selective disruption of phosphatidylcholine metabolism of the intracellular parasite Toxoplasma gondii arrests its growth. J Biol Chem. 2005;280(16):16345-53; doi:10.1074/jbc.M501523200. https://www.ncbi.nlm.nih.gov/pubmed/15708856.

31. Serran-Aguilera L, Denton H, Rubio-Ruiz B, Lopez-Gutierrez B, Entrena A, Izquierdo L, et al. Plasmodium falciparum Choline Kinase Inhibition Leads to a Major Decrease in Phosphatidylethanolamine Causing Parasite Death. Sci Rep. 2016;6:33189; doi:10.1038/srep33189.

https://www.ncbi.nlm.nih.gov/pubmed/27616047.

32. Stirnimann CU, Petsalaki E, Russell RB, Muller CW. WD40 proteins propel cellular networks. Trends Biochem Sci. 2010;35 10:565-74; doi:10.1016/j.tibs.2010.04.003. https://www.ncbi.nlm.nih.gov/pubmed/20451393.

33. von Bohl A, Kuehn A, Simon N, Ngongang VN, Spehr M, Baumeister S, et al. A WD40-repeat protein unique to malaria parasites associates with adhesion protein complexes and is crucial for blood stage progeny. Malar J. 2015;14:435; doi:10.1186/s12936-015-0967-x. https://www.ncbi.nlm.nih.gov/pubmed/26537493.

34. Jing H, Takagi J, Liu JH, Lindgren S, Zhang RG, Joachimiak A, et al. Archaeal surface layer proteins contain beta propeller, PKD, and beta helix domains and are related to metazoan cell surface proteins. Structure. 2002;10 10:1453-64; doi:10.1016/s0969-2126(02)00840-7.

https://www.ncbi.nlm.nih.gov/pubmed/12377130.

35. Tosini F, Agnoli A, Mele R, Gomez Morales MA, Pozio E. A new modular protein of Cryptosporidium parvum, with ricin B and LCCL domains, expressed in the sporozoite invasive stage. Mol Biochem Parasitol. 2004;134(1):137-47; doi:10.1016/j.molbiopara.2003.11.014.

https://www.ncbi.nlm.nih.gov/pubmed/14747151.

36. Rosenzweig R, Nillegoda NB, Mayer MP, Bukau B. The Hsp70 chaperone network. Nat Rev Mol Cell Biol. 2019;20(11):665-80. doi:10.1038/s41580019-0133-3. https://pubmed.ncbi.nIm.nih.gov/31253954.

37. Miller CM, Akratos C, Johnson AM, Smith NC. The production of a 70 kDa heat shock protein by Toxoplasma gondii RH strain in immunocompromised mice. Int J Parasitol. 2000;30 14:1467 - 1273; doi: 10.1016/s0020-7519(00)00118-1.

https://www.ncbi.nlm.nih.gov/pubmed/11428337.

38. Khramtsov NV, Tilley M, Blunt DS, Montelone BA, Upton SJ. Cloning and analysis of a Cryptosporidium parvum gene encoding a protein with homology to cytoplasmic form Hsp70. J Eukaryot Microbiol. 1995;42(4):416-22; doi:10.1111/j.1550-7408.1995.tb01605.x.

https://www.ncbi.nlm.nih.gov/pubmed/7620467.

39. Woods KM, Tilley M, Iseli A, Upton SJ, Montelone BA, Khramtsov NV. Sequence of the gene encoding hsp90e from Cryptosporidium parvum. DNA SEq. 1999;10(4-5):339-42; doi:10.3109/10425179909033961. https://www.ncbi.nlm.nih.gov/pubmed/10727089.

40. Lyons RE, Johnson AM. Heat shock proteins of Toxoplasma gondii. Parasite Immunol. 1995;17 7:353-9; doi:10.1111/j.1365-3024.1995.tb00902.x. https://www.ncbi.nlm.nih.gov/pubmed/8552407.

\section{Tables}

Table 1 Upregulated and downregulated proteins after excystation of Cryptosporidium andersoni oocysts 


\begin{tabular}{|c|c|c|c|c|c|c|c|}
\hline Type & Description & Accession no. & Coverage & Proteins AAs & $\begin{array}{l}\mathrm{MW} \\
(\mathrm{kDa})\end{array}$ & $\begin{array}{l}\text { Ratio } \\
\text { (Excysted/Non- } \\
\text { excysted) }\end{array}$ & P-value \\
\hline \multirow[t]{10}{*}{ Upregulated } & 40s ribosomal protein S25 & A0A1J4MSM1 & 59.4 & 7 & 11.685 & 1.264 & 0.00091609 \\
\hline & Ethanolaminephosphotransferase & A0A1J4MTE1 & 2.7 & 1 & 43.263 & 1.258 & 0.00040461 \\
\hline & Histone $\mathrm{H} 2 \mathrm{~A}$ & A0A1J4MVP1 & 16.8 & 3 & 14.623 & 1.375 & 0.00043533 \\
\hline & $\begin{array}{l}\text { Regulator of chromosome } \\
\text { condensation (RCC1) repeat } \\
\text { family protein }\end{array}$ & A0A2P4Z0T8 & 0.5 & 1 & 212.46 & 3.52 & 0.0000011953 \\
\hline & Uncharacterized protein & A0A1J4M9W2 & 3.3 & 2 & 64.459 & 1.326 & 0.0097439 \\
\hline & Uncharacterized protein & A0A1J4MHV2 & 8.8 & 1 & 15.025 & 1.384 & 0.0064401 \\
\hline & Uncharacterized protein & A0A1J4MNM9 & 19.4 & 3 & 14.569 & 1.207 & 0.000299 \\
\hline & Uncharacterized protein & A0A1J4MP75 & 7.3 & 2 & 25.649 & 1.221 & 0.020465 \\
\hline & Uncharacterized protein & A0A1J4MT89 & 15.1 & 4 & 35.279 & 1.289 & 0.0003197 \\
\hline & Uncharacterized protein & B6AJJ3 & 2.7 & 1 & 38.258 & 1.345 & 0.0117384 \\
\hline \multirow[t]{7}{*}{ Downregulated } & Aspartyl protease family protein & A0A1J4MIX1 & 16.6 & 15 & 88.828 & 0.833 & 0.000063593 \\
\hline & $\begin{array}{l}\text { Glycosyl transferase family } 8 \\
\text { protein }\end{array}$ & A0A1J4MU57 & 6.1 & 4 & 55.147 & 0.769 & 0.0020419 \\
\hline & Putative rRNA methyltransferase & A0A2P4YX32 & 0.8 & 1 & 115.75 & 0.764 & 0.0007558 \\
\hline & $\begin{array}{l}\text { Putative integral membrane } \\
\text { protein }\end{array}$ & A0A2P4YZ96 & 0.2 & 1 & 738.34 & 0.813 & 0.0047774 \\
\hline & Thioredoxin reductase & A0A1J4MPC1 & 7.3 & 4 & 55.183 & 0.734 & 0.000043038 \\
\hline & Uncharacterized protein & A0A0S4TFF5 & 0.1 & 1 & 1516.4 & 0.477 & $1.71 \mathrm{E}-05$ \\
\hline & Uncharacterized protein & A0A1J4MAX1 & 3.2 & 1 & 31.311 & 0.764 & 0.0003441 \\
\hline
\end{tabular}

\section{Figures}




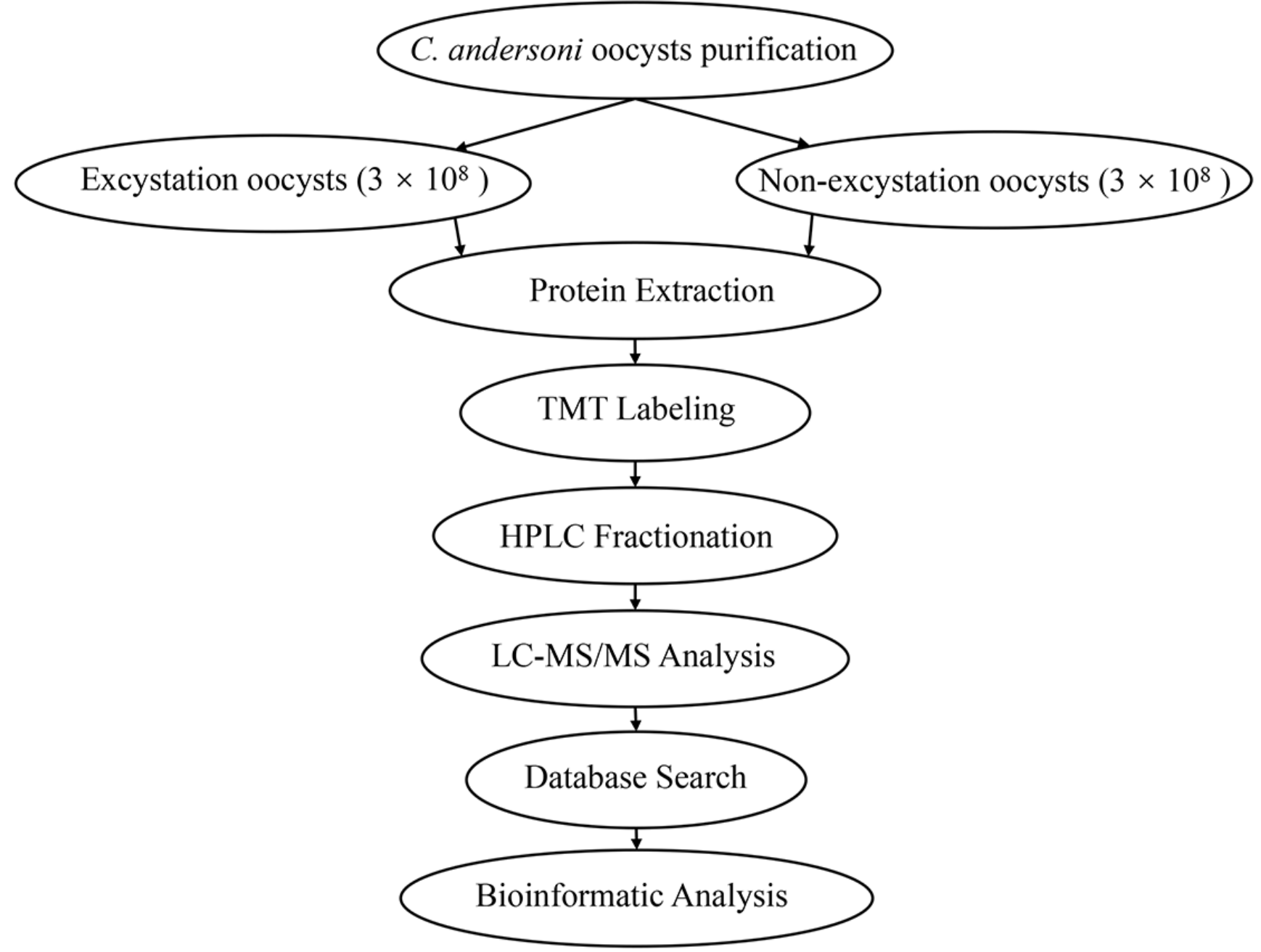

Figure 1

To understand how the proteome changes with Cryptosporidium andersoni oocyst excystation, a TMT-based labeling approach coupled with High Performance Liquid Chromatography (HPLC) and LC-MS/MS was employed to identify the proteome of excysted and non-excysted oocysts (Fig. 1). 

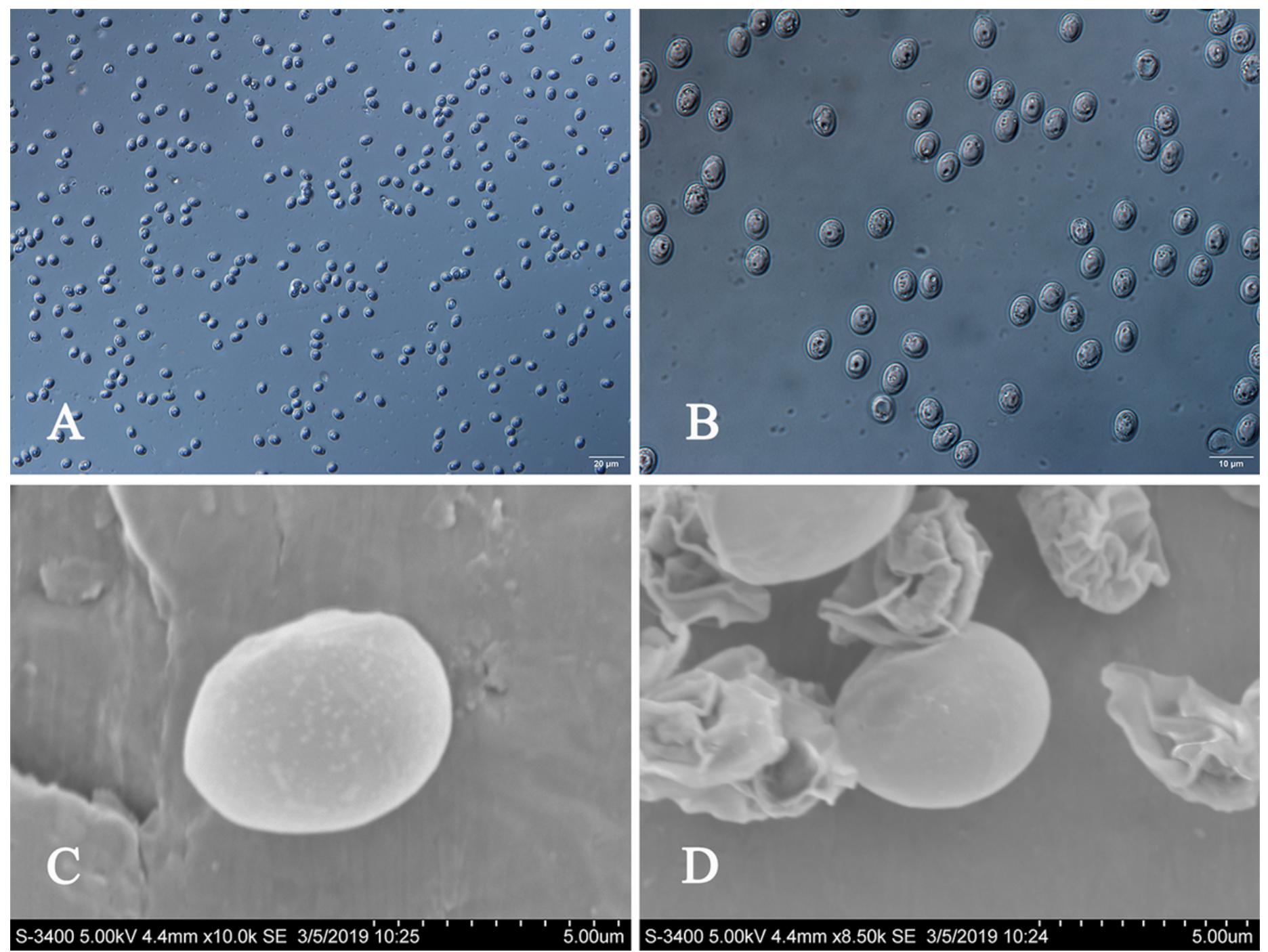

S-3400 5.00kV 4.4mm x10.0k SE 3/5/2019'10:25'

\section{Figure 2}

Highly purified oocysts of $\mathrm{C}$. andersoni were obtained from adult cow's feces via sucrose solution density gradient centrifugation and cesium chloride density gradient centrifugation (Figure 2). 


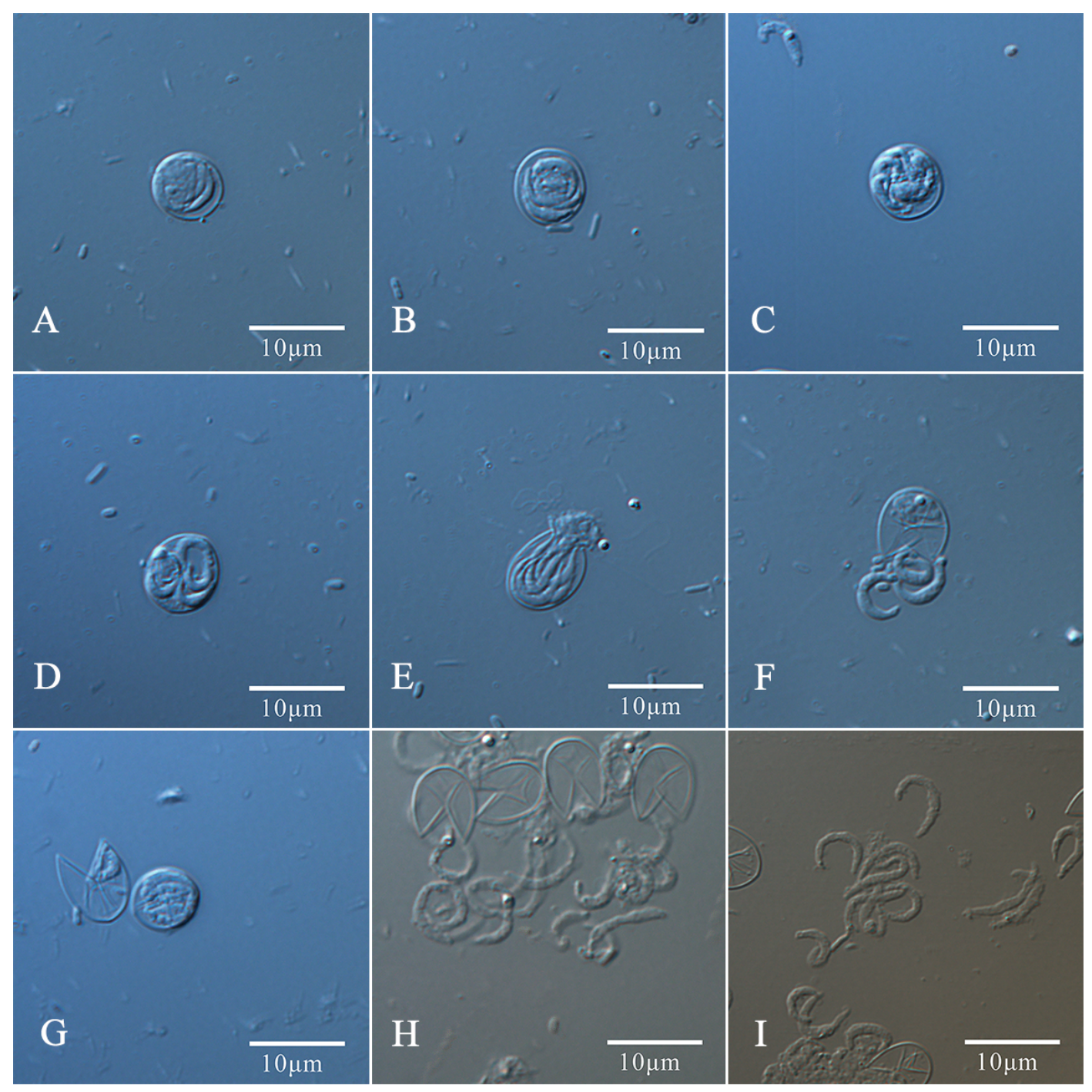

\section{Figure 3}

The excystation rate of C. andersoni was $82 \%$ using 3-h incubation in a $37^{\circ} \mathrm{C}$ thermostat water bath. Non-excysted oocysts, excysted oocysts, and sporozoites are shown in Figure 3. 
A

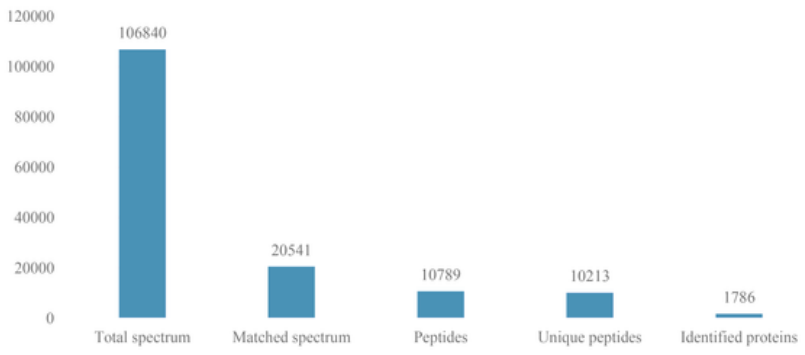

B

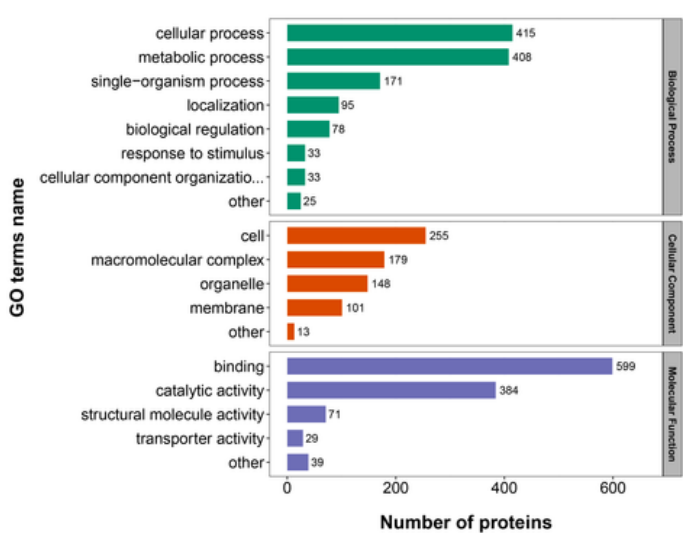

C

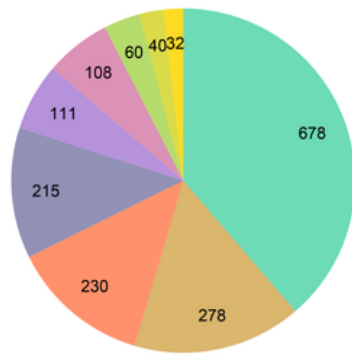

cytoplasm (38.7\%)

plasma membrane $(15.87 \%)$

extracellular $(13.13 \%)$

mitochondria (6.34\%)

cytoplasm,nucleus (6.16\%)

cytoskeleton (3.42\%)

other $(1.83 \%)$
D

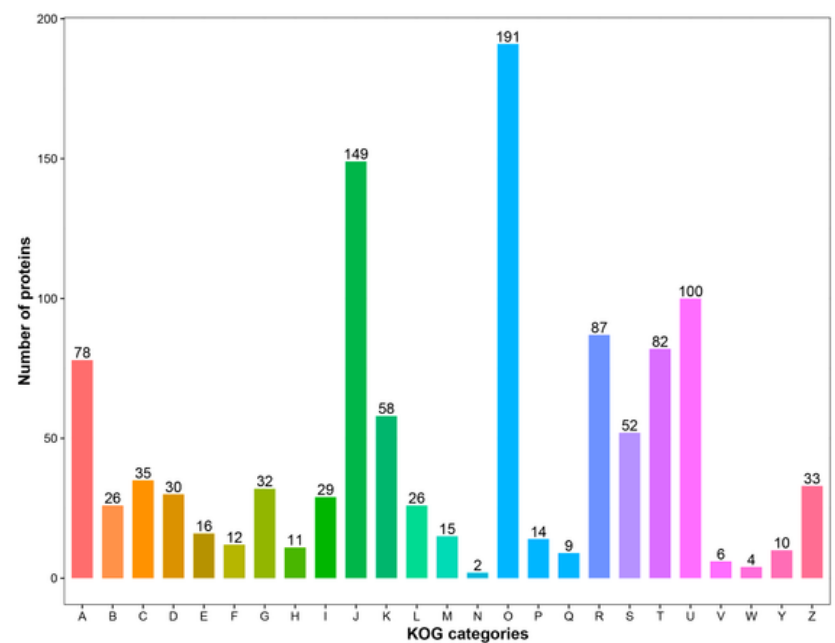

nucleus (12.27\%)

endoplasmic reticulum $(2.28 \%)$

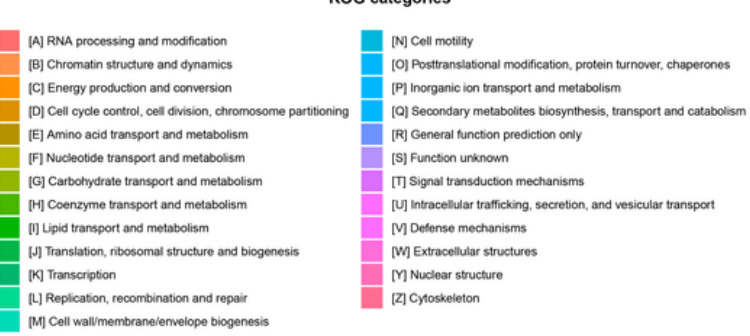

\section{Figure 4}

A subset of 20541 effective spectra were obtained after the mass spectrometry two-level graph was searched by the protein theory data, and the spectral graph utilization rate was $19.2 \%$. A total of 10789 peptide segments were identified by spectral analysis, with 10213 unique peptide segments, and 1786 proteins were identified (Fig. 4). 
A

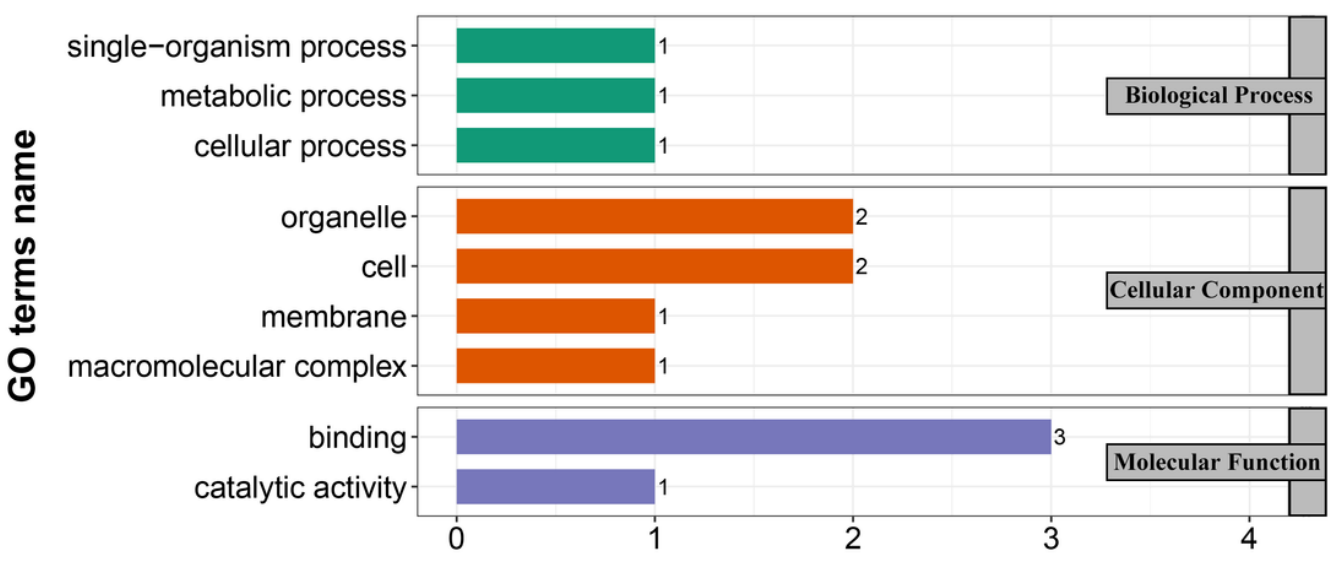

Number of proteins

B

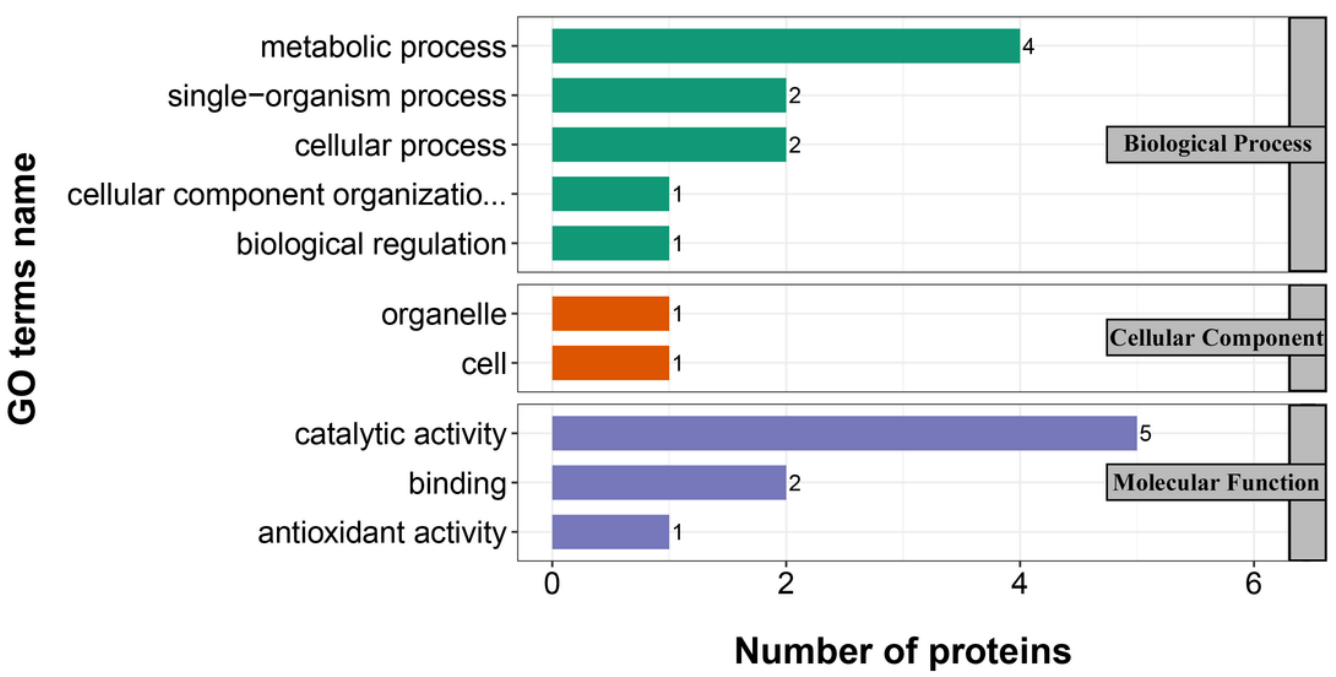

Figure 5

We used a 1.2-fold increase or decrease in protein expression as a benchmark for physiologically significant changes to determine the proteins that were differentially expressed after excystation. In total, 17 DEPs were identified between excysted and non-excysted oocysts. Among these DEPs, 10 were upregulated and 7 were downregulated after C. andersoni excystation (Table S3). According to the GO enrichment analysis (Fig. 5), 13, 8, and 12 proteins were enriched for biological process, cell component, and molecular function, respectively. Subcellular localization predictions showed that the DEPs were located in the cytoplasm (4), plasma membrane (3), nucleus (3), extracellular (2), mitochondria (2), cytoplasm or nucleus (1), and cytoskeleton (1) (Fig. 5). 
A

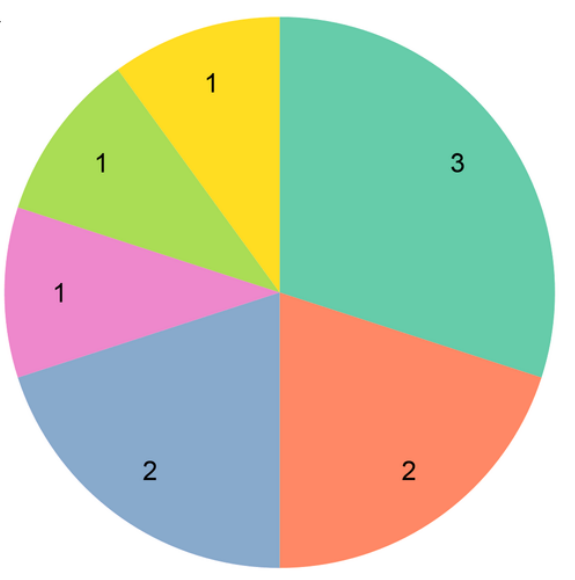

cytoplasm (30\%)

nucleus (20\%)

mitochondria (20\%)

cytoplasm,nucleus (10\%)

plasma membrane (10\%)

cytoskeleton (10\%)

B

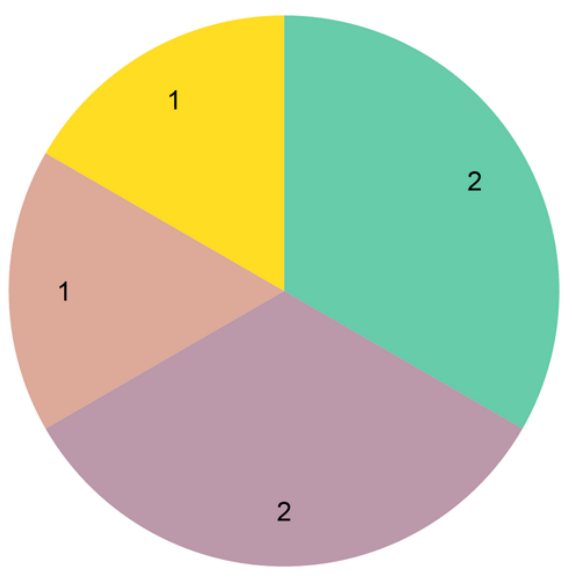

extracellular (33.33\%)

plasma membrane (33.33\%)

nucleus $(16.67 \%)$

cytoplasm (16.67\%)

\section{Figure 6}

Functional enrichment analyses of the DEPs (Fig. 5) suggested that the DEPs were significantly enriched for "RNA processing and modification" (2), "posttranslational modification, protein turnover, chaperones" (2), "general function prediction only" (2), "chromatin structure and dynamics" (1), "energy production and conversion" (1), "amino acid transport and metabolism" (1), "lipid transport and metabolism" (1), and "translation, ribosomal structure and biogenesis" (1). Unfortunately, these proteins were not enriched into any KEGG pathway. In addition, we focused on upregulated proteins after excystation of C. andersoni oocysts. The unregulated proteins were significantly enriched in "binding" (3) and were mainly localized in the cytoplasm (3), nucleus (2), and mitochondria (2) (Fig. 6), suggesting that upregulated proteins were mainly associated with control of gene expression at the level of transcription, for example RCC1, Histone H2A, and an unidentified protein (ID: A0A1J4MP75). 

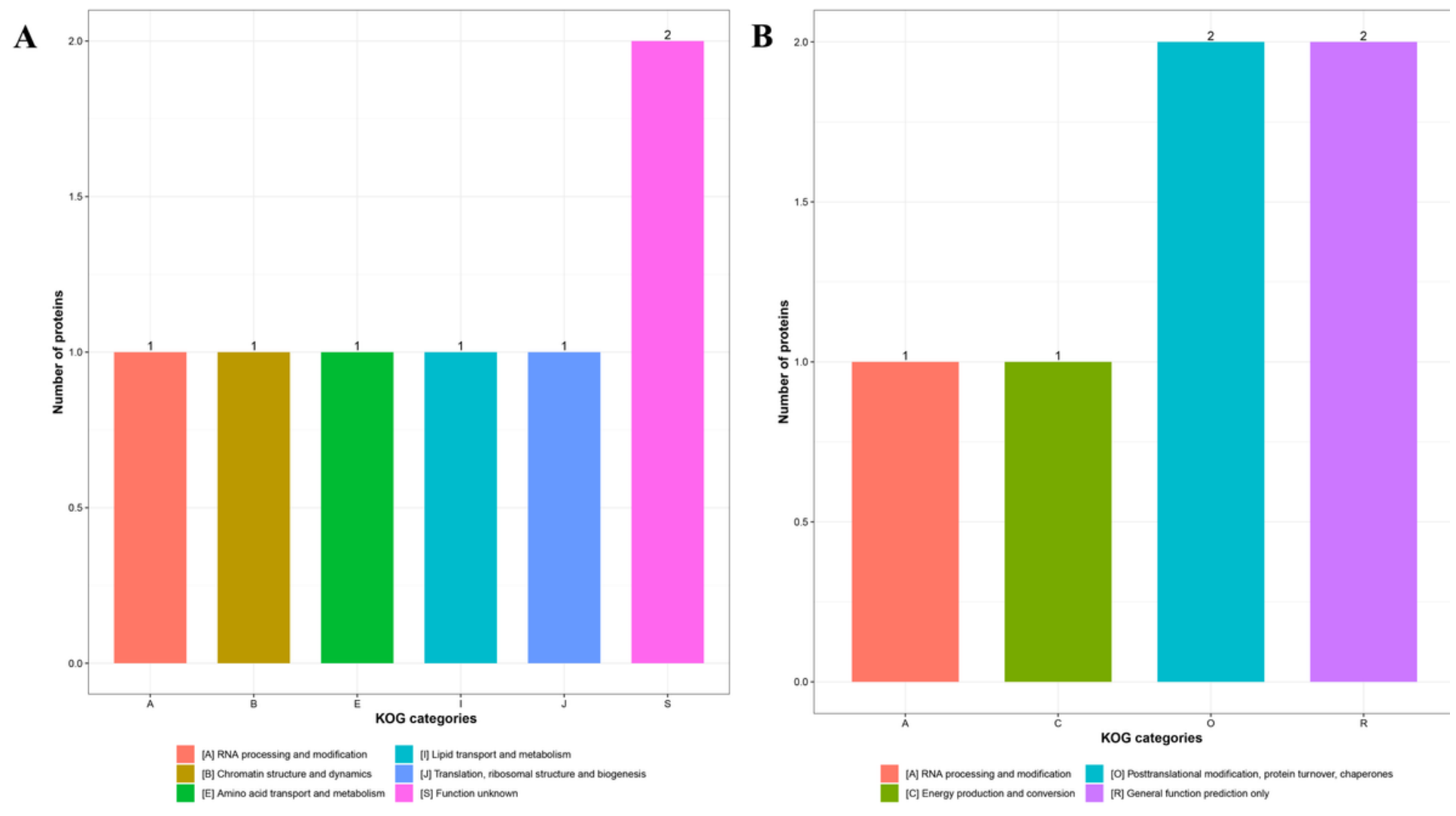

Figure 7

To investigate the excystation mechanism, the transcription levels of DEPs were verified by qRT-PCR. Nine DEPs were selected randomly for qRT-PCR analysis to characterize gene expression. The qRT-PCR results indicated that the transcription levels of most genes were consistent with expression levels according to TMT data (Fig. 7).

\section{Supplementary Files}

This is a list of supplementary files associated with this preprint. Click to download.

- Graphicalabstract.tif

- Tables1.docx

- Tables2MSidentifiedinformation.xIsx

- TableS3Differentiallyexpressedstatistics.xIsx 